\title{
Existence for Time-Fractional Semilinear Diffusion Equation on the Sphere
}

\author{
N. D. Phuong, ${ }^{1}$ Ho Duy Binh, ${ }^{2}$ Ho Thi Kim Van, ${ }^{2}$ and Le Dinh Long $\mathbb{D}^{2}$ \\ ${ }^{1}$ Faculty of Fundamental Science, Industrial University of Ho Chi Minh City, Ho Chi Minh City 700000, Vietnam \\ ${ }^{2}$ Division of Applied Mathematics, Thu Dau Mot University, Binh Duong Province, Vietnam \\ Correspondence should be addressed to Le Dinh Long; ledinhlong@tdmu.edu.vn
}

Received 1 May 2021; Accepted 28 May 2021; Published 24 June 2021

Academic Editor: Mustafa Inc

Copyright (C) 2021 N. D. Phuong et al. This is an open access article distributed under the Creative Commons Attribution License, which permits unrestricted use, distribution, and reproduction in any medium, provided the original work is properly cited.

Fractional diffusion on the sphere plays a large role in the study of physical phenomena customs and meteorology and geophysics. In this paper, we examine two types of the sphere problem: the initial value problem and the end value problem. We are interested in focus on the solution existence in a local or global form. In order to overcome difficult evaluations when evaluating, we need some new techniques. The main analytical tool is the use of the Banach fixed point theorem.

\section{Introduction}

When examining many physical and geophysical phenomena, one encounters problems directly or indirectly related to the sphere. To describe and explain quantitatively these models, they will be simulated with mathematical equations on the sphere. That is also the reason why the spherical equations have attracted many scientists interested and studied them. We can give some examples as follows. The weather forecasting models and the currents of groundwater in the ocean bed were simulated by equations on the sphere. For the readers' convenience, we have given a number of typical works that have had a great influence on the development of the analysis of PDEs on the sphere.

The qualitative and numerical methods have been considered by many authors, such as Thong et al. [1,2]. Cauchy problems for elliptical queries on spheres have been studied in $[3,4]$. The Navier-Stokes equation on the $2 \mathrm{D}$ unit sphere has been considered by the recent paper [5]. Recently, pseudo-parabolic equation on the sphere has been studied in [6]. Intuitively, we realize that the root structure of the differential equation on the sphere can be complex, so studying the types of partial derivative equations on the sphere requires mathematical tools with many new techniques.
According to the development of the mathematics disciplines, especially calculus, in the last few decades, fractional analysis has been one of the most influential disciplines in mathematics. Most of the problems related to it often have applications in modeling real-world problems. Fraction analysis has many applications in mechanics, physics, engineering science, etc. We would like to share many published works on these issues such as Karapinar et al. [7-14] and Inc and his group [15-19].

The main goal of this paper is to investigate the existence of the following equation

$$
\frac{\partial}{\partial t} u(x, t)-\frac{\partial^{1-\alpha}}{\partial t} \Delta^{*} u(x, t)=\theta(t) F(u(x, t)),(x, t) \in S^{2} \times[0, T],
$$

with the initial Cauchy condition

$$
u(x, 0)=f(x)
$$

or the terminal value condition

$$
u(x, T)=g(x)
$$


where $\partial^{\alpha} / \partial t^{\alpha}$ called the Riemann-Liouville fractional derivative of order $\alpha, 0<\alpha \leq 1$. Here, $S^{2}$ is a sphere on the $R^{3}$. It is given by $\partial^{1-\alpha} / \partial t$ is the Riemann-Liouville fractional derivative of order $1-\alpha$ given by

$$
\frac{\partial^{1-\alpha}}{\partial t} v(t):=\frac{1}{\Gamma(\alpha)} \frac{d}{d t}\left(\int_{0}^{t} s^{\alpha-1} v(t-s) d s\right), 0<\alpha<1,
$$

and $D_{0^{+}}^{0.02 \mathrm{~cm} \alpha} v(t)=:(d / d t) v(t)$ if $\alpha=1$. The functions $\psi, F$ in (1) are defined later. The operator $\Delta^{*}$ is called LaplaceBeltrami which is introduced in more detail in Section 2. To the best of our knowledge, there are not any results on problem (1). Our main goal in this paper is to study two goals. Our first goal is to consider Cauchy initial problem (1)-(2). We get the global solution in a suitable space. Our second goal is to consider the Cauchy terminal problem (1)-(2). In this case, we get only local solutions in space $L^{\infty}$ . For both of the above purposes, we use Banach fixed point theorem together with the evaluation of the sphere. In addition, to overcome the difficulties of proofing, we also cleverly make use of subtle evaluations of the Mittag-Leffler functions. The two main difficulties shown are as follows:

(i) First, we deal with spherical harmonics on the sphere and require complex calculations

(ii) Second, we must make sharp judgments for the Mittag-Leffler functions to achieve our goal

This paper is structured as follows. In Section 2, we introduce some preliminaries on Mittag-Leffler functions and their properties, Sobolev space on the sphere, and some other spaces. In Section 3, we focus on the initial value problem for problem (1)-(2). Section 4 provides the local well-posed result for terminal value problem (1)-(3).

\section{Preliminaries}

Mittag-Leffler is a function represented as the following form

$$
E_{\alpha, \beta}(\xi)=\sum_{n=0}^{\infty} \frac{\xi^{n}}{\Gamma(n \alpha+\beta)},
$$

$(\xi \in \mathbb{C})$, for $\alpha>0$ and $\beta \in \mathbb{R}$. We call to mind the following lemmas (see for example [20]). We have the following lemma which is useful for next proof.

Lemma 1. Let $0<\alpha<1$. Then, the function $E_{\alpha, 1}(-z)$ satisfies the following property

$$
\frac{C_{1}}{1+z} \leq E_{\alpha, 1}(-z) \leq \frac{C_{2}}{1+z}, z>0,
$$

where $C_{1}$ and $C_{2}$ are the two positive constants.

Spherical harmonics are polynomials which satisfy $\Delta_{x} Y($ $x)=0$ (where $\Delta_{x}$ is the Laplacian operator in $R^{3}$ ) and are restricted to the surface of the Euclidean sphere $S^{2}$. The eigenvalues for $-\Delta^{*}$ in $R^{3}$ are

$$
\lambda_{j}=j^{2}+j, j=0,1,2, \cdots \cdots,
$$

and the eigenfunctions corresponding to $\lambda_{j}$ are the spherical harmonics $\mathbf{W}_{j}(x)$ of order l, i.e.,

$$
\Delta^{*} \mathbf{W}_{j}(x)=-\lambda_{j} \mathbf{W}_{j}(x)
$$

The space of all spherical harmonics of degree $j$ on $S^{2}$, denoted by $V_{j}$, has an orthonormal basis $\left\{\mathbf{W}_{j k}(x): k=1,2,3\right.$, $\cdots \mathcal{N}(2, j)\}$ where

$$
\mathcal{N}(2,0)=1, \mathcal{N}(2, j)=\frac{2 j+1}{\Gamma(2)}, j \geq 1 .
$$

Let any function $f \in L^{2}\left(S^{2}\right)$, so it is expressed by the expansion of spherical harmonics

$$
f=\sum_{j=0}^{\infty} \sum_{k=1}^{(2 j+1) / \Gamma(2)} \widehat{f}_{j k} W_{j k}, \widehat{f}_{j k}=\int_{S^{2}} f \overline{\mathbf{W}}_{j k} d S
$$

where dS is the surface measure of the unit sphere. The Sobolev space $\mathbf{H}^{v}\left(S^{2}\right)$ is defined by

$$
\mathbf{H}^{v}\left(S^{2}\right)=\left\{g \in L^{2}\left(S^{2}\right): \sum_{j=0}^{\infty} \sum_{k=1}^{(2 j+1) / \Gamma(2)}\left(j^{2}+j+1\right)^{v}\left|g \wedge_{j k}\right|^{2}<\infty\right\},
$$

with the following norm

$$
\|g\|_{\mathbf{H}^{v}\left(S^{2}\right)}=\sqrt{\sum_{j=0}^{\infty} \sum_{j=1}^{(2 j+1) / \Gamma(2)}\left(j^{2}+j+1\right)^{v}\left|g \wedge_{j k}\right|^{2}} .
$$

The space $L_{a}^{\infty}\left(0, T ; \mathbf{H}^{v}\left(S^{2}\right)\right)$ is defined by

$$
L_{a}^{\infty}\left(0, T ; \mathbf{H}^{v}\left(S^{2}\right)\right)=\left\{f \in L^{\infty}\left(0, T ; \mathbf{H}^{v}\left(S^{2}\right)\right), e^{-a t}\|f(., t)\|_{\mathbf{H}^{v}\left(S^{2}\right)}<\infty\right\},
$$

with corresponding norm as follows

$$
\|f\|_{L_{a}^{\infty}\left(0, T ; \mathbf{H}^{v}\left(S^{2}\right)\right)}=\sup _{0 \leq t \leq T} e^{-a t}\|f(., t)\|_{\mathbf{H}^{v}\left(S^{2}\right)} .
$$

\section{Global Existence for Mild Solution to Initial Value Problem}

Theorem 2. Assume that $\theta:(0, T) \longrightarrow \mathbb{R}$ such that

$$
|\theta(z)| \leq C_{\theta} z^{m},-\frac{1}{2}<m .
$$


Let $F$ satisfies the condition

$$
\left\|F v_{1}-F v_{2}\right\|_{\mathbf{H}^{v^{\prime}}\left(s^{2}\right)} \leq K_{f}\left\|v_{1}-v_{2}\right\|_{\mathbf{H}^{v}\left(s^{2}\right)},
$$

where $K_{f}$ is a positive constant and $v$ and $v^{\prime}$ satisfy that

$$
0<v-v^{\prime}<\min \left(2, \frac{1}{\alpha}\right)
$$

Then, problem (1)-(2) has a global existence in $L_{a}^{\infty}(0, T$ $\left.; \mathbf{H}^{v}\left(S^{2}\right)\right)$ for a enough large.

Proof. As we know from [2] that $\Delta^{*}$ is the Laplace-Beltrami on the sphere $S^{2}$. Any function $u \in L^{2}\left(S^{2}\right)$ can be described by the terms of spherical harmonics

$$
\begin{aligned}
u(x, t) & =\sum_{j=0}^{\infty} \sum_{k=1}^{(2 j+1) / \Gamma(2)} \widehat{u}_{j k}(t) \mathbf{W}_{j k}(x), \widehat{u}_{j k}(t) \\
& =\int_{S^{2}} u(x, t) \overline{\mathbf{W}}_{j k}(x) d S,
\end{aligned}
$$

where $d S$ is the surface measure of the unit sphere. Let us first give an expression of the mild solution.

$$
\begin{aligned}
\widehat{u}_{j k}(t)= & E_{\alpha, 1}\left(-\left(j^{2}+j\right) t^{\alpha}\right) \widehat{f}_{j k} \\
& +\int_{0}^{t} E_{\alpha, 1}\left(-\left(j^{2}+j\right)(t-r)^{\alpha}\right) \theta(r) \widehat{F}_{j k}(u)(r) d r .
\end{aligned}
$$

So, we get that the following equality

$$
\begin{aligned}
u(x, t)= & \sum_{j=0}^{\infty} \sum_{k=1}^{(2 j+1) / \Gamma(2)}\left(E_{\alpha, 1}\left(-\left(j^{2}+j\right) t^{\alpha}\right) \widehat{f}_{j k}\right) \mathbf{W}_{j k}(x)+\sum_{j=0}^{\infty} \sum_{k=1}^{(2 j+1) / \Gamma(2)} \\
& \cdot\left(\int_{0}^{t} E_{\alpha, 1}\left(-\left(j^{2}+j\right)(t-r)^{\alpha}\right) \theta(r) \widehat{F}_{j k}(u)(r) d r\right) \mathbf{W}_{j k}(x) .
\end{aligned}
$$

Set the following function

$$
\begin{aligned}
\mathscr{J} w(t)= & \sum_{j=0}^{\infty} \sum_{k=1}^{(2 j+1) / \Gamma(2)}\left(E_{\alpha, 1}\left(-\left(j^{2}+j\right) t^{\alpha}\right) \widehat{f}_{j k}\right) \mathbf{W}_{j k}(x)+\sum_{j=0}^{\infty} \sum_{k=1}^{(2 j+1) / \Gamma(2)} \\
& \cdot\left(\int_{0}^{t} E_{\alpha, 1}\left(-\left(j^{2}+j\right)(t-r)^{\alpha}\right) \theta(r) \widehat{F}_{j k}(w)(r) d r\right) \mathbf{W}_{j k} .
\end{aligned}
$$

Setting the function

$$
\mathscr{M}_{1}(x, t)=\sum_{j=0}^{\infty} \sum_{k=1}^{(2 j+1) / \Gamma(2)}\left(E_{\alpha, 1}\left(-\left(j^{2}+j\right) t^{\alpha}\right) \widehat{f}_{j k}\right) \mathbf{W}_{j k}(x)
$$

Noting that $E_{\alpha, 1}(-z) \leq C_{2}$ for any $z>0$, and $f \in \mathbf{H}^{v}\left(S^{2}\right)$, the first term is bounded by

$$
\begin{aligned}
\left\|\mathscr{M}_{1}(., t)\right\|_{\mathbf{H}^{v}\left(S^{2}\right)}^{2} & =\sum_{j=0}^{\infty} \sum_{k=1}^{(2 j+1) / \Gamma(2)}\left(j^{2}+j+1\right)^{v}\left(E_{\alpha, 1}\left(-\left(j^{2}+j\right) t^{\alpha}\right) f \wedge_{j k}\right)^{2} \\
& \leq\left|C_{2}\right|^{2} \sum_{l=0}^{\infty} \sum_{k=1}^{(2 j+1) / \Gamma(2)}\left(j^{2}+j+1\right)^{v}\left|f \wedge_{l k}\right|^{2} \\
& =\left|C_{2}\right|^{2}\|f\|_{\mathbf{H}^{v}\left(S^{2}\right)}^{2}
\end{aligned}
$$

which allows us to get that

$$
\left\|\mathscr{M}_{1}(., t)\right\|_{\mathbf{H}^{v}\left(s^{2}\right)} \leq C_{2}\|f\|_{\mathbf{H}^{v}\left(s^{2}\right)},
$$

for any $0 \leq t \leq T$. This gives immediately that if $w=0$ then $\mathscr{J} w$ belongs to the space $L^{\infty}\left(0, T ; \mathbf{H}^{v}\left(S^{2}\right)\right)$. Let us take two functions $w$ and $\bar{w}$ belong to the space $L^{\infty}\left(0, T ; \mathbf{H}^{v}\left(S^{2}\right.\right.$ )). Then, from (21), we have

$$
\begin{aligned}
\mathscr{J} w(t)-\mathscr{J} \bar{w}(t)= & \sum_{j=0}^{\infty} \sum_{k=1}^{(2 j+1) / \Gamma(2)}\left(\int _ { 0 } ^ { t } E _ { \alpha , 1 } \left(-\left(j^{2}+j\right)\right.\right. \\
& \left.\left.\cdot(t-r)^{\alpha}\right) \theta(r)\left(\widehat{F}_{j k}(w)(r)-\widehat{F}_{j k}(\bar{w})(r)\right) d r\right) \mathbf{W}_{j k}(x) .
\end{aligned}
$$

Using Parseval's equality and Hölder inequality, one has

$$
\begin{aligned}
\| \mathscr{J} w( & t)-\mathscr{J} \bar{w}(t) \|_{\mathbf{H}^{v}\left(S^{2}\right)}^{2} \\
= & \sum_{j=0}^{\infty} \sum_{k=1}^{(2 j+1) / \Gamma(2)}\left(j^{2}+j+1\right)^{v} \\
& \cdot\left(\int_{0}^{t} E_{\alpha, 1}\left(-\left(j^{2}+j\right)(t-r)^{\alpha}\right) \theta(r)\right. \\
& \left.\cdot\left(F \wedge_{j k}(w)(r)-F \wedge_{j k}(\bar{w})(r)\right) d r\right)^{2} \\
\leq & \sum_{j=0}^{\infty} \sum_{k=1}^{(2 j+1) / \Gamma(2)}\left(j^{2}+j+1\right)^{v} \\
& \cdot\left(\int_{0}^{t}\left|E_{\alpha, 1}\left(-\left(j^{2}+j\right)(t-r)^{\alpha}\right) \theta(r)\right|^{2} d r\right) \\
& \cdot\left(\int_{0}^{t}\left(F \wedge_{j k}(w)(r)-F \wedge_{j k}(\bar{w})(r)\right)^{2} d r\right) .
\end{aligned}
$$

Let us review that $E_{\alpha, 1}(-z) \leq\left(C_{2} /(1+z)\right)$, we note that the following inequality 


$$
E_{\alpha, 1}\left(-\left(j^{2}+j\right) t^{\alpha}\right) \leq \frac{C_{2}}{1+\left(j^{2}+j\right) t^{\alpha}} \leq C_{2}\left(j^{2}+j\right)^{-\beta} t^{-\alpha \beta}
$$

for $0<\beta<1$. Noting that $j^{2}+j \geq\left(\left(j^{2}+j+1\right) / 2\right)$ we get that the following inequality

$$
\begin{aligned}
& \left(j^{2}+j+1\right)^{\nu}\left(\int_{0}^{t}\left|E_{\alpha, 1}\left(-\left(j^{2}+j\right)(t-r)^{\alpha}\right) \theta(r)\right|^{2} d r\right) \\
& \leq\left|C_{2}\right|^{2}\left|C_{\theta}\right|^{2} 4^{-\beta}\left(j^{2}+j+1\right)^{\nu-2 \beta} \int_{0}^{t}(t-r)^{-2 \alpha \beta} r^{2 m} d r .
\end{aligned}
$$

Since the assumption $1-2 \alpha \beta>0$ and $1+2 m>0$, we can deduce that

$$
\int_{0}^{t}(t-r)^{-2 \alpha \beta} r^{2 m} d r=t^{1-2 \alpha \beta+2 m} \mathbf{B}(1-2 \alpha \beta, 1+2 m)
$$

Here, $\mathbf{B}$ is beta function. Let us choose $\beta=1 / 2\left(v-v^{\prime}\right)$; we see that $\beta$ satisfies $1-2 \alpha \beta>0$. Combining (26) and (28), we provide that

$$
\begin{aligned}
\|\mathscr{J} w(t)-\mathscr{J} \bar{w}(t)\|_{\mathbf{H}^{v}\left(s^{2}\right)}^{2} \leq & \left|\tilde{C}_{1}\right|^{2} \sum_{j=0}^{\infty} \sum_{k=1}^{(2 j+1) / \Gamma(2)}\left(j^{2}+j+1\right)^{v-2 \beta} \\
& \cdot\left(\int_{0}^{t}\left(F \wedge_{j k}(w)(r)-F \wedge_{j k}(\bar{w})(r)\right)^{2}\right) \\
= & \left|\tilde{C}_{1}\right|^{2} \int_{0}^{t}\|F(w)(r)-F(\bar{w})(r)\|_{\mathbf{H}^{v-2 \beta}\left(s^{2}\right)}^{2} d r \\
= & \left|\tilde{C}_{1}\right|^{2} \int_{0}^{t}\|F(w)(r)-F(\bar{w})(r)\|_{\mathbf{H}^{v^{\prime}}\left(s^{2}\right)}^{2} d r,
\end{aligned}
$$

where we set

$$
\left|\tilde{C}_{1}\right|^{2}=\left|C_{2}\right|^{2}\left|C_{\theta}\right|^{2} 4^{-\beta} \mathbf{B}(1-2 \alpha \beta, 1+2 m) .
$$
$a>0$

Thanks to Lipschitz property of $\mathscr{J}$, we find that for any

$$
\begin{aligned}
& e^{-2 a t}\|\mathscr{J} w(t)-\mathscr{J} \bar{w}(t)\|_{\mathbf{H}^{v}\left(s^{2}\right)}^{2} \\
& \quad \leq\left|\tilde{C}_{1}\right|^{2} K_{f}^{2} T^{1-2 \alpha \beta+2 m} \int_{0}^{t} e^{-2 a(t-r)} e^{-2 a r}\|w(r)-\bar{w}(r)\|_{\mathbf{H}^{v}\left(S^{2}\right)}^{2} d r \\
& \quad \leq\left|\tilde{C}_{1}\right|^{2} K_{f}^{2} T^{1-2 \alpha \beta+2 m}\left(\int_{0}^{t} e^{-2 a(t-r)} d r\right)\|w-\bar{w}\|_{L_{a}^{\infty}\left(0, T ; \mathbf{H}^{v}\left(S^{2}\right)\right)}^{2}
\end{aligned}
$$

Due to the condition $\int_{0}^{t} e^{-2 a(t-r)} d r \leq(1 / 2 a)$, we know that the following estimate

$$
\begin{aligned}
& e^{-2 a t}\|\mathscr{J} w(t)-\mathscr{J} \bar{w}(t)\|_{\mathbf{H}^{v}\left(s^{2}\right)}^{2} \\
& \quad \leq \frac{\left|\tilde{C}_{1}\right|^{2} K_{f}^{2} T^{1-2 \alpha \beta+2 m}}{2 a}\|w-\bar{w}\|_{L_{a}^{\infty}\left(0, T ; \mathbf{H}^{v}\left(S^{2}\right)\right)}^{2}
\end{aligned}
$$

This implies immediately that

$$
e^{-a t}\|\mathscr{J} w(t)-\mathscr{J} \bar{w}(t)\|_{H^{v}\left(S^{2}\right)} \leq \sqrt{\frac{\left.\left.\right|^{\sim} C_{1}\right|^{2} K_{f}^{2} T^{1-2 \alpha \beta+2 m}}{2 a\|w-\bar{w}\|_{L_{a}^{\infty}\left(0, T ; \mathbf{H}^{v}\left(S^{2}\right)\right)}}}
$$

The right hand side of (34) is independent of $t$, so we can deduce that the following estimate

$\|\mathscr{J} w-\mathscr{J} \bar{w}\|_{L_{a}^{\infty}\left(0, T ; \mathbf{H}^{v}\left(S^{2}\right)\right)} \leq \sqrt{\frac{\left.\tilde{C}_{1}\right|^{2} K_{f}^{2} T^{1-2 \alpha \beta+2 m}}{2 a}}\|w-\bar{w}\|_{L_{a}^{\infty}\left(0, T ; \mathbf{H}^{v}\left(S^{2}\right)\right)}$.

By choose $a$ enough large such that $\sqrt{\left(\left.\tilde{C}_{1}\right|^{2} K_{f}^{2} T^{1-2 \alpha \beta+2 m} / 2 a\right)}<1$, we find that $\mathscr{J}$ is a contraction in $L_{a}^{\infty}\left(0, T ; \mathbf{H}^{v}\left(S^{2}\right)\right)$. Based on the Banach fixed point theorem, we have immediately concluded that problem (1)-(2) has a global existence in $L_{a}^{\infty}\left(0, T ; \mathbf{H}^{v}\left(S^{2}\right)\right)$.

\section{Terminal Value Problem: Local Existence}

In this section, we devoted the following problem with terminal condition

$$
\begin{cases}\frac{\partial}{\partial t} w(x, t)-\frac{\partial^{1-\alpha}}{\partial t} \Delta^{*} w(x, t)=\theta(t) F(u(x, t)), & (x, t) \in S^{2} \times(0, T), \\ w(x, T)=g(x), & x \in S^{2},\end{cases}
$$

where $g$ is defined later. The purpose of this section is to study the existence and uniqueness of solution of problem (1)-(3).

Theorem 3. Let us assume that $\psi:(0, T) \longrightarrow \mathbb{R}$ such that

$$
|\theta(z)| \leq C_{\theta} z^{\delta}, \delta>-1
$$

Let the function $g \in \mathbf{H}^{2}\left(S^{2}\right)$. Let $F$ satisfies the condition

$$
\left\|F v_{1}-F v_{2}\right\|_{L^{2}\left(S^{2}\right)} \leq K_{f}\left\|v_{1}-v_{2}\right\|_{L^{2}\left(S^{2}\right)}
$$


for any $K_{f}>0$. Then, problem (1)-(3) has a local existence in $L^{\infty}\left(0, T ; L^{2}\left(S^{2}\right)\right)$.

Proof. Let us assume that $u(x, 0)=f(x)$. Then, we have

$$
\begin{aligned}
\widehat{w}_{j k}(t)= & E_{\alpha, 1}\left(-\left(j^{2}+j\right) t^{\alpha}\right) \widehat{f}_{j k} \\
& +\int_{0}^{t} E_{\alpha, 1}\left(-\left(j^{2}+j\right)(t-r)^{\alpha}\right) \theta(r) \widehat{F}_{j k}(w)(r) d r .
\end{aligned}
$$

Set $t=T$ into the above equation, we get that

$$
\begin{aligned}
\widehat{w}_{j k}(T)= & E_{\alpha, 1}\left(-\left(j^{2}+j\right) T^{\alpha}\right) \widehat{f}_{j k} \\
& +\int_{0}^{T} E_{\alpha, 1}\left(-\left(j^{2}+j\right)(T-r)^{\alpha}\right) \theta(r) \widehat{F}_{j k}(w)(r) d r .
\end{aligned}
$$

This implies that

$$
\begin{aligned}
\widehat{f}_{j k}= & \frac{1}{E_{\alpha, 1}\left(-\left(j^{2}+j\right) T^{\alpha}\right)} \\
& \cdot\left(\widehat{g}_{j k}-\int_{0}^{T} E_{\alpha, 1}\left(-\left(j^{2}+j\right)(T-r)^{\alpha}\right) \theta(r) \widehat{F}_{j k}(w)(r) d r\right) .
\end{aligned}
$$

After some simple calculation, we have that

$$
\begin{aligned}
\widehat{w}_{j k}(t)= & \frac{E_{\alpha, 1}\left(-\left(j^{2}+j\right) t^{\alpha}\right)}{E_{\alpha, 1}\left(-\left(j^{2}+j\right) T^{\alpha}\right)} \widehat{f}_{j k} \\
& +\int_{0}^{t} E_{\alpha, 1}\left(-\left(j^{2}+j\right)(t-r)^{\alpha}\right) \theta(r) \widehat{F}_{j k}(w)(r) d r \\
& -\frac{E_{\alpha, 1}\left(-\left(j^{2}+j\right) t^{\alpha}\right)}{E_{\alpha, 1}\left(-\left(j^{2}+j\right) T^{\alpha}\right)} \int_{0}^{T} E_{\alpha, 1} \\
& \cdot\left(-\left(j^{2}+j\right)(T-r)^{\alpha}\right) \theta(r) \widehat{F}_{j k}(w)(r) d r .
\end{aligned}
$$

So, we get that the following equality

$$
\begin{aligned}
w(x, t)= & \sum_{j=0}^{\infty} \sum_{k=1}^{(2 j+1) / \Gamma(2)}\left(\frac{E_{\alpha, 1}\left(-\left(j^{2}+j\right) t^{\alpha}\right)}{E_{\alpha, 1}\left(-\left(j^{2}+j\right) T^{\alpha}\right)} \widehat{g}_{j k}\right) \mathbf{W}_{j k}(x) \\
& +\sum_{j=0}^{\infty} \sum_{k=1}^{(2 j+1) / \Gamma(2)}\left(\int _ { 0 } ^ { t } E _ { \alpha , 1 } \left(-\left(j^{2}+j\right)\right.\right. \\
& \left.\left.\cdot(t-r)^{\alpha}\right) \theta(r) \widehat{F}_{j k}(w)(r) d r\right) \mathbf{W}_{j k}(x) \\
& -\sum_{j=0}^{\infty} \sum_{k=1}^{(2 j+1) / \Gamma(2)}\left(\frac{E_{\alpha, 1}\left(-\left(j^{2}+j\right) t^{\alpha}\right)}{E_{\alpha, 1}\left(-\left(j^{2}+j\right) T^{\alpha}\right)} \int_{0}^{T} E_{\alpha, 1}\right. \\
& \left.\cdot\left(-\left(j^{2}+j\right)(T-r)^{\alpha}\right) \theta(r) \widehat{F}_{j k}(w)(r) d r\right) \mathbf{W}_{j k}(x) .
\end{aligned}
$$

In order to apply Banach fixed point theorem, we need to set the following function

$$
\begin{aligned}
\mathscr{T} \theta(t)= & \underbrace{\sum_{j=0}^{\infty} \sum_{k=1}^{(2 j+1) / \Gamma(2)}\left(\frac{E_{\alpha, 1}\left(-\left(j^{2}+j\right) t^{\alpha}\right)}{E_{\alpha, 1}\left(-\left(j^{2}+j\right) T^{\alpha}\right)} \widehat{g}_{j k}\right) \mathbf{W}_{j k}}_{\mathscr{T}_{0}(t)} \\
& +\underbrace{\sum_{j=0}^{\infty} \sum_{k=1}^{(2 j+1) / \Gamma(2)}\left(\int_{0}^{t} E_{\alpha, 1}\left(-\left(j^{2}+j\right)(t-r)^{\alpha}\right) \theta(r) \widehat{F}_{j k}(\theta)(r) d r\right) \mathbf{W}_{j k}}_{\mathscr{T}_{1} \theta(t)} \\
& -\underbrace{\underbrace{}_{\mathscr{T}_{2} \theta(t)}}_{\sum_{j=0}^{\infty} \sum_{k=1}^{(2 j+1) / \Gamma(2)}\left(\frac{E_{\alpha, 1}\left(-\left(j^{2}+j\right) t^{\alpha}\right)}{E_{\alpha, 1}\left(-\left(j^{2}+j\right) T^{\alpha}\right)} \int_{0}^{T} E_{\alpha, 1}\left(-\left(j^{2}+j\right)(T-r)^{\alpha}\right) \theta(r) \widehat{F}_{j k}(\theta)(r) d r\right) \mathbf{W}_{j k}} .
\end{aligned}
$$

First, let us look at the expression as above and give an evaluation for $\left\|\mathscr{T}_{2} \theta_{1}(t)-\mathscr{T}_{2} \theta_{2}(t)\right\|$ for any $\theta_{1}, \theta_{2} \in L^{\infty}(0$, $\left.T ; L^{2}\left(S^{2}\right)\right)$. First, noting that

$$
\begin{gathered}
E_{\alpha, 1}\left(-\left(j^{2}+j\right) T^{\alpha}\right) \geq \frac{C_{1}}{1+\left(j^{2}+j\right) T^{\alpha}}, \\
E_{\alpha, 1}\left(-\left(j^{2}+j\right)(T-r)^{\alpha}\right) \leq \frac{C_{2}}{1+\left(j^{2}+j\right)(T-r)^{\alpha}},
\end{gathered}
$$


we get that

$$
\begin{aligned}
\frac{E_{\alpha, 1}\left(-\left(j^{2}+j\right)(T-r)^{\alpha}\right)}{E_{\alpha, 1}\left(-\left(j^{2}+j\right) T^{\alpha}\right)} & \leq \frac{C_{2}}{C_{1}} \frac{1+\left(j^{2}+j\right) T^{\alpha}}{1+\left(j^{2}+j\right)(T-r)^{\alpha}} \\
& \leq C_{3} T^{\alpha}(T-r)^{-\alpha} .
\end{aligned}
$$

This implies immediately that

$$
\begin{aligned}
\| \mathscr{T}_{2} & \theta_{1}(t)-\mathscr{T}_{2} \theta_{2}(t) \|_{L^{2}\left(S^{2}\right)}^{2} \\
= & \sum_{j=0}^{\infty} \sum_{k=1}^{(2 j+1) / \Gamma(2)}\left(\frac{E_{\alpha, 1}\left(-\left(j^{2}+j\right) t^{\alpha}\right)}{E_{\alpha, 1}\left(-\left(j^{2}+j\right) T^{\alpha}\right)}\right. \\
& \cdot \int_{0}^{T} E_{\alpha, 1}\left(-\left(j^{2}+j\right)(T-r)^{\alpha}\right) \theta(r) \\
& \left.\cdot\left(F \wedge_{j k}\left(\theta_{1}\right)(r)-F \wedge_{j k}\left(\theta_{2}\right)(r)\right) d r\right)^{2} \\
\leq & \sum_{j=0}^{\infty} \sum_{k=1}^{(2 j+1) / \Gamma(2)}\left(\int_{0}^{T} E_{\alpha, 1}\left(-\left(j^{2}+j\right)(T-r)^{\alpha}\right)\right. \\
& \left.\cdot \frac{E_{\alpha, 1}\left(-\left(j^{2}+j\right) t^{\alpha}\right)}{E_{\alpha, 1}\left(-\left(j^{2}+j\right) T^{\alpha}\right)} \theta(r) d r\right)\left(\int_{0}^{T} E_{\alpha, 1}\left(-\left(j^{2}+j\right)(T-r)^{\alpha}\right)\right. \\
& \left.\cdot \frac{E_{\alpha, 1}\left(-\left(j^{2}+j\right) t^{\alpha}\right)}{E_{\alpha, 1}\left(-\left(j^{2}+j\right) T^{\alpha}\right)} \theta(r)\left(F \wedge_{j k}\left(\theta_{1}\right)(r)-F \wedge_{j k}\left(\theta_{2}\right)(r)\right)^{2} d r\right),
\end{aligned}
$$

where we have used Hölder inequality. Using (46), we have that

$$
\begin{gathered}
\int_{0}^{T} E_{\alpha, 1}\left(-\left(j^{2}+j\right)(T-r)^{\alpha}\right) \frac{E_{\alpha, 1}\left(-\left(j^{2}+j\right) t^{\alpha}\right)}{E_{\alpha, 1}\left(-\left(j^{2}+j\right) T^{\alpha}\right)} \theta(r) d r \\
\leq C_{3} C_{1} T^{\alpha} C_{\theta} \int_{0}^{T}(T-r)^{-\alpha} r^{\delta} d r \\
=C_{3} C_{1} T^{\alpha} C_{\theta} T^{1-\alpha+\delta} \mathbf{B}(1-\alpha, 1+\delta) \\
=C_{3} C_{1} C_{\theta} T^{1+\delta} \mathbf{B}(1-\alpha, 1+\delta), \\
\int_{0}^{T} E_{\alpha, 1}\left(-\left(j^{2}+j\right)(T-r)^{\alpha}\right) \frac{E_{\alpha, 1}\left(-\left(j^{2}+j\right) t^{\alpha}\right)}{E_{\alpha, 1}\left(-\left(j^{2}+j\right) T^{\alpha}\right)} \theta(r)\left(F \wedge_{j k}\left(\theta_{1}\right)(r)-F \wedge_{j k}\left(\theta_{2}\right)(r)\right)^{2} d r \\
\leq C_{3} C_{1} T^{\alpha} C_{\theta} \int_{0}^{T}(T-r)^{-\alpha} r^{\delta}\left(F \wedge_{j k}\left(\theta_{1}\right)(r)-F \wedge_{j k}\left(\theta_{2}\right)(r)\right)^{2} d r .
\end{gathered}
$$

Hence, we can deduce that

$$
\begin{aligned}
\| \mathscr{T}_{2} & \theta_{1}(t)-\mathscr{T}_{2} \theta_{2}(t) \|_{L^{2}\left(s^{2}\right)}^{2} \\
\leq & \left|C_{3} C_{1} C_{\theta}\right|^{2} T^{1+\delta+\alpha} \mathbf{B}(1-\alpha, 1+\delta) \\
\quad & \quad \int_{0}^{T}(T-r)^{-\alpha} r^{\delta}\left\|F\left(\theta_{1}\right)(r)-F\left(\theta_{2}\right)(r)\right\|_{L^{2}\left(s^{2}\right)}^{2} d r .
\end{aligned}
$$

Lipschitz property of $F$ as in (38) gives that

$$
\begin{aligned}
& \int_{0}^{T}(T-r)^{-\alpha} r^{\delta}\left\|F\left(\theta_{1}\right)(r)-F\left(\theta_{2}\right)(r)\right\|_{L^{2}\left(S^{2}\right)}^{2} d r \\
& \quad \leq K_{f} \int_{0}^{T}(T-r)^{-\alpha} r^{\delta}\left\|\theta_{1}(., r)-\theta_{2}(., r)\right\|_{L^{2}\left(S^{2}\right)}^{2} d r \\
& \quad \leq K_{f}\left(\int_{0}^{T}(T-r)^{-\alpha} r^{\delta} d r\right)\left\|\theta_{1}-\theta_{2}\right\|_{L^{\infty}\left(0, T ; L^{2}\left(S^{2}\right)\right)}^{2} \\
& \quad=K_{f} T^{1-\alpha+\delta} \mathbf{B}(1-\alpha, 1+\delta)\left\|\theta_{1}-\theta_{2}\right\|_{L^{\infty}\left(0, T ; L^{2}\left(S^{2}\right)\right)}^{2}
\end{aligned}
$$

Combining (49) and (50), we find that

$$
\begin{aligned}
& \left\|\mathscr{T}_{2} \theta_{1}(t)-\mathscr{T}_{2} \theta_{2}(t)\right\|_{L^{2}\left(s^{2}\right)}^{2} \\
& \quad \leq\left|C_{3} C_{1} C_{\theta}\right|^{2} T^{2+2 \delta}|\mathbf{B}(1-\alpha, 1+\delta)|^{2}\left\|\theta_{1}-\theta_{2}\right\|_{L^{\infty}\left(0, T ; L^{2}\left(s^{2}\right)\right)}^{2},
\end{aligned}
$$

which allows us to get that

$$
\begin{aligned}
& \left\|\mathscr{T}_{2} \theta_{1}(t)-\mathscr{T}_{2} \theta_{2}(t)\right\|_{L^{2}\left(s^{2}\right)} \\
& \quad \leq C_{3} C_{1} C_{\theta} T^{1+\delta} \mathbf{B}(1-\alpha, 1+\delta)\left\|\theta_{1}-\theta_{2}\right\|_{L^{\infty}\left(0, T ; L^{2}\left(s^{2}\right)\right)} .
\end{aligned}
$$

The term to the right of above expression is independent of $t$, so we get that

$$
\begin{aligned}
& \left\|\mathscr{T}_{2} \theta_{1}-\mathscr{T}_{2} \theta_{2}\right\|_{L^{\infty}\left(0, T ; L^{2}\left(S^{2}\right)\right)} \\
& \quad \leq C_{3} C_{1} C_{\theta} T^{1+\delta} \mathbf{B}(1-\alpha, 1+\delta)\left\|\theta_{1}-\theta_{2}\right\|_{L^{\infty}\left(0, T ; L^{2}\left(S^{2}\right)\right)} .
\end{aligned}
$$

By a similar argument as above, we deduce that

$$
\begin{aligned}
\| \mathscr{T}_{1} & \theta_{1}(t)-\mathscr{T}_{1} \theta_{2}(t) \|_{L^{2}\left(s^{2}\right)}^{2} \\
= & \sum_{j=0}^{\infty} \sum_{k=1}^{(2 j+1) / \Gamma(2)}\left(\int_{0}^{t} E_{\alpha, 1}\left(-\left(j^{2}+j\right)(t-r)^{\alpha}\right) \theta(r)\right. \\
& \left.\cdot\left(F \wedge_{j k}\left(\theta_{1}\right)(r)-F \wedge_{j k}\left(\theta_{2}\right)(r)\right) d r\right)^{2} \\
\leq & \sum_{j=0}^{\infty} \sum_{k=1}^{(2 j+1) / \Gamma(2)}\left(\int_{0}^{t} E_{\alpha, 1}\left(-\left(j^{2}+j\right)(t-r)^{\alpha}\right) \theta(r) d r\right) \\
& \cdot\left(\int_{0}^{t} E_{\alpha, 1}\left(-\left(j^{2}+j\right)(t-r)^{\alpha}\right) \theta(r)\right. \\
& \left.\cdot\left(F \wedge_{j k}\left(\theta_{1}\right)(r)-F \wedge_{j k}\left(\theta_{2}\right)(r)\right)^{2} d r\right),
\end{aligned}
$$

where in the last inequality, we have used Hölder inequality. Let us repeat that $E_{\alpha, 1}\left(-\left(j^{2}+j\right)(t-r)^{\alpha}\right) \leq C_{2},(14)$ and $\theta(r) \leq$ $C_{\theta} r^{\delta}$, and thanks to that, we immediately have the following two estimations 


$$
\begin{aligned}
& \int_{0}^{t} E_{\alpha, 1}\left(-\left(j^{2}+j\right)(t-r)^{\alpha}\right) \theta(r) d r \\
& \quad \leq C_{2} C_{\theta} \int_{0}^{t} r^{\delta} d r=C_{2} C_{\theta} \frac{t^{1+\delta}}{1+\delta} \leq \frac{C_{2} C_{\theta} T^{1+\delta}}{1+\delta} .
\end{aligned}
$$

Combining (54) and (55), we arrive at

$$
\begin{aligned}
& \left\|\mathscr{T}_{1} \theta_{1}(t)-\mathscr{T}_{1} \theta_{2}(t)\right\|_{L^{2}\left(s^{2}\right)}^{2} \\
& \leq \frac{C_{2} C_{\theta} T^{1+\delta}}{1+\delta} C_{2} C_{\theta} \int_{0}^{t} r^{\delta}\left\|F\left(\theta_{1}\right)(r)-F\left(\theta_{2}\right)(r)\right\|_{L^{2}\left(s^{2}\right)}^{2} d r \\
& \leq \frac{K_{f}^{2} C_{2} C_{\theta} T^{1+\delta}}{1+\delta} C_{2} C_{\theta} \int_{0}^{t} r^{\delta}\left\|\theta_{1}(., r)-\theta_{2}(., r)\right\|_{L^{2}\left(s^{2}\right)}^{2} d r \\
& \leq \frac{K_{f}^{2} C_{2} C_{\theta} T^{1+\delta}}{1+\delta} C_{2} C_{\theta}\left(\int_{0}^{t} r^{\delta} d r\right)\left\|\theta_{1}-\theta_{2}\right\|_{L^{\infty}}^{2}\left(0, T ; L^{2}\left(s^{2}\right)\right) \\
& \leq\left(\frac{K_{f} C_{2} C_{\theta} T^{1+\delta}}{1+\delta}\right)^{2} \mathbf{B}(1-\alpha, 1+\delta)\left\|\theta_{1}-\theta_{2}\right\|_{L^{\infty}\left(0, T ; L^{2}\left(s^{2}\right)\right)}^{2} .
\end{aligned}
$$

The right hand side of (56) is independent of $t$; we have the following conclusion immediately

$$
\begin{aligned}
& \left\|\mathscr{T}_{1} \theta_{1}-\mathscr{T}_{1} \theta_{2}\right\|_{L^{\infty}\left(0, T ; L^{2}\left(S^{2}\right)\right)} \\
& \quad \leq \frac{K_{f} C_{2} C_{\theta} T^{1+\delta}}{1+\delta} \sqrt{\mathbf{B}(1-\alpha, 1+\delta)}\left\|\theta_{1}-\theta_{2}\right\|_{L^{\infty}\left(0, T ; L^{2}\left(S^{2}\right)\right)} .
\end{aligned}
$$

From these two assertions (44), (53), and (57) and using the triangle inequality, we have the following affirmation

$$
\begin{aligned}
\| & \mathscr{T} \theta_{1}-\mathscr{T} \theta_{2} \|_{L^{\infty}}\left(0, T ; L^{2}\left(S^{2}\right)\right) \\
\leq & \left\|\mathscr{T}_{1} \theta_{1}-\mathscr{T}_{1} \theta_{2}\right\|_{L^{\infty}}\left(0, T ; L^{2}\left(S^{2}\right)\right) \\
& +\left\|\mathscr{T}_{2} \theta_{1}-\mathscr{T}_{2} \theta_{2}\right\|_{L^{\infty}}\left(0, T ; L^{2}\left(S^{2}\right)\right) \\
\leq & C_{3} C_{1} C_{\theta} T^{1+\delta} \mathbf{B}(1-\alpha, 1+\delta)\left\|\theta_{1}-\theta_{2}\right\|_{L^{\infty}}\left(0, T ; L^{2}\left(S^{2}\right)\right) \\
& \quad+\frac{K_{f} C_{2} C_{\theta} T^{1+\delta}}{1+\delta} \sqrt{\mathbf{B}(1-\alpha, 1+\delta)} \| \theta_{1} \\
& -\theta_{2} \|_{L^{\infty}}\left(0, T ; L^{2}\left(S^{2}\right)\right) .
\end{aligned}
$$

By choose $T$ enough small, we can conclude that $\mathscr{T}$ is a contraction. Next, we need to check that $\mathscr{T}_{0}(t) g \in L^{\infty}(0, T$ ; $\left.L^{2}\left(S^{2}\right)\right)$. Indeed, we get for any $0 \leq t \leq T$

$$
\begin{aligned}
\left\|\mathscr{T}_{0}(t) g\right\|_{L^{2}\left(S^{2}\right)}^{2} & =\sum_{j=0}^{\infty} \sum_{k=1}^{(2 j+1) / \Gamma(2)}\left(\frac{E_{\alpha, 1}\left(-\left(j^{2}+j\right) t^{\alpha}\right)}{E_{\alpha, 1}\left(-\left(j^{2}+j\right) T^{\alpha}\right)}\right)^{2}\left|g \wedge_{j k}\right|^{2} \\
& \leq \sum_{j=0}^{\infty} \sum_{k=1}^{(2 j+1) / \Gamma(2)}\left(\frac{1+\left(j^{2}+j\right) T^{\alpha}}{C_{1}}\right)^{2}\left|f \wedge_{j k}\right|^{2} \\
& \leq \tilde{C}\|g\|_{\mathbf{H}^{2}\left(S^{2}\right)}^{2} .
\end{aligned}
$$

Using Banach fixed point theorem, we can conclude that $\mathscr{T}$ has a fixed point $w$ in $L^{\infty}\left(0, T ; L^{2}\left(S^{2}\right)\right)$. Hence, we get the desired result.

\section{Conclusion}

In this paper, this is one of our first results about fractional diffusion on the sphere. In this article, we are interested in the existing existence in local form and global format with the main tool is the Banach fixed point theorem. In the future, we study the ill-posedness of this problem and show the convergent rate between the sought solution and the regularized solution.

\section{Data Availability}

No data were used to support this study.

\section{Conflicts of Interest}

The authors declare that they have no competing interests.

\section{Authors' Contributions}

Both authors contributed equally and significantly in writing this paper. Four authors read and approved the final manuscript.

\section{Acknowledgments}

This research is supported by the Industrial University of Ho Chi Minh City (IUH) under grant number 66/HD-DHCN.

\section{References}

[1] Q. T. L. Gia, N. H. Tuan, and T. Tran, "Solving the backward heat equation on the unit sphere," ANZIAM Journal, vol. 56, pp. C262-C278, 2016.

[2] Q. T. Le Gia, "Approximation of parabolic PDEs on spheres using spherical basis functions," Advances in Computational Mathematics, vol. 22, no. 4, pp. 377-397, 2005.

[3] Q. T. Le Gia, "Galerkin approximation for elliptic PDEs on spheres," Journal of Approximation Theory, vol. 130, no. 2, pp. 125-149, 2004.

[4] Q. T. Le Gia, I. H. Sloan, and T. Tran, "Overlapping additive Schwarz preconditioners for elliptic PDEs on the unit sphere," Mathematics of Computation, vol. 78, no. 265, pp. 79-101, 2009.

[5] Z. Brzeźniak, B. Goldys, and Q. T. Le Gia, "Random attractors for the stochastic Navier-Stokes equations on the $2 \mathrm{D}$ unit sphere," Journal of Mathematical Fluid Mechanics, vol. 20, no. 1, pp. 227-253, 2018.

[6] N. D. Phuong and N. H. Luc, "Note on a nonlocal pseudoparabolic equation on the unit sphere," Dynamic Systems and Applications, vol. 30, no. 2, pp. 295-304, 2019.

[7] R. S. Adiguzel, U. Aksoy, and E. Karapinar, "New anisotropic models from isotropic solutions," Mathematical Methods in the Applied Sciences, vol. 29, no. 1, pp. 67-83, 2006.

[8] H. Afshari and E. Karapnar, "A discussion on the existence of positive solutions of the boundary value problems via-Hilfer 
fractional derivative on b-metric spaces," Advances in Difference Equations, vol. 2020, no. 1, 11 pages, 2020.

[9] H. Afshari, S. Kalantari, and E. Karapinar, "Solution of fractional differential equations via coupled fixed point," Electronic Journal of Differential Equations, vol. 2015, no. 1, pp. 1-12, 2015.

[10] B. Alqahtani, H. Aydi, E. Karapnar, and V. Rakocevic, "A solution for Volterra fractional integral equations by hybrid contractions," Mathematics, vol. 7, no. 8, p. 694, 2019.

[11] E. Karapinar, A. Fulga, M. Rashid, L. Shahid, and H. Aydi, "Large contractions on quasi-metric spaces with an application to nonlinear fractional differential-equations," Mathematics, vol. 7, no. 5, p. 444, 2019.

[12] A. Salim, B. Benchohra, E. Karapinar, and J. E. Lazreg, "Existence and Ulam stability for impulsive generalized Hilfertype fractional differential equations," Advances in Difference Equations, vol. 2020, 21 pages, 2020.

[13] E. Karapinar, T. Abdeljawad, and F. Jarad, "Applying new fixed point theorems on fractional and ordinary differential equations," Advances in Difference Equations, vol. 2019, 25 pages, 2019.

[14] A. Abdeljawad, R. P. Agarwal, E. Karapinar, and P. S. Kumari, "Solutions of the nonlinear integral equation and fractional differential equation using the technique of a fixed point with a numerical experiment in extended b-metric space," Symmetry, vol. 11, no. 5, p. 686, 2019.

[15] A. Yusuf, B. Acay, U. T. Mustapha, M. Inc, and D. Baleanu, "Mathematical modeling of pine wilt disease with Caputo fractional operator," Chaos, Solitons and Fractals, vol. 143, p. 110569, 2021.

[16] B. Acay and M. Inc, "Fractional modeling of temperature dynamics of a building with singular kernels," Chaos, Solitons and Fractals, vol. 142, p. 110482, 2021.

[17] Z. Korpinar, M. Inc, and M. Bayram, "Theory and application for the system of fractional Burger equations with Mittag leffler kernel," Applied Mathematics and Computation, vol. 367, p. 124781, 2020.

[18] X. J. Yang, Y. Y. Feng, C. Cattani, and M. Inc, "Fundamental solutions of anomalous diffusion equations with the decay exponential kernel," Mathematicsl Methods in the Applied Sciences, vol. 42, no. 11, pp. 4054-4060, 2019.

[19] M. S. Hashemi, E. Darvishi, and M. Inc, "A geometric numerical integration method for solving the Volterra integrodifferential equations," International Journal of Computer Mathematics, vol. 95, no. 8, pp. 1654-1665, 2018.

[20] I. Podlubny, Fractional Differential Equations, Academic Press, London, 1999. 